\title{
Review of: "The deleterious effects induced by an acute exposure of human skin to common air pollutants are prevented by extracts of Deschampsia antarctica"
}

\section{khan hira}

Potential competing interests: The author(s) declared that no potential competing interests exist.

1. So, the extract of Deschampsia antarctica is producing blocking/protectant effect against polluted environmental conditions from air pollutants. If this is the case, then it could be used as pollutant block just like the sun block in formuation/cosmetic product in the form of lotion or cream.

2. Author can perform the phytochemical screening of individual phyto-constituents like alkaloids, flavonoids, glycosides, phenols, lignins, saponins, sterols, tannins, anthraquinone, and reducing sugar and determine that how much percentage of each individual antioxidant is present in the extract of Deschampsia antarctica

3. Author also can discuss the effects of Deschampsia antarctica extract about the UVR, ozone, nitrogen dioxide and sulphur dioxde as they also produce deleterious effects on skin as pollutants. 